\title{
Blood rheology during normal pregnancy
}

\author{
Panagiotis Tsikouras ${ }^{\mathrm{c}}$, Barbara Niesigk $^{\mathrm{b}}$, Georg-Friedrich von Tempelhoff ${ }^{\mathrm{a}, *}$, Werner Rath ${ }^{\mathrm{e}}$, \\ Olga Schelkunov ${ }^{\mathrm{a}}$, Peter Daragód and Roland Csorba ${ }^{\mathrm{b}, \mathrm{d}}$ \\ ${ }^{a}$ Department of Obstetrics and Gynecology, Vinzenz Hospital of Hanau, Germany \\ ${ }^{\mathrm{b}}$ Department of Obstetrics and Gynecology, City Hospital of Aschaffenburg, Aschaffenburg, Germany \\ ${ }^{\mathrm{c}}$ Department of Obstetrics and Gynecology, Democritus University of Thrace, Alexandroupolis, Greece \\ ${ }^{\mathrm{d} D e p a r t m e n t}$ of Obstetrics and Gynecology, Faculty of Medicine, University of Debrecen, Hungary \\ ${ }^{\mathrm{e}}$ Department of Obstetrics and Gynecology University of Schleswig-Holstein Kiel, Germany
}

\begin{abstract}
.
INTRODUCTION: Recent studies have shown increased RBC aggregation and no difference in plasma viscosity in the presence of markedly lower hematocrit in women at term compared to non-pregnant women. Little is known about the outcome of blood rheological parameters and red blood cell (RBC) deformability particularly in the course of normal pregnancy.

METHODS: During a 36 months interval 1.913 blood samples were randomly collected from a total of 945 pregnant women in the course of their pregnancy $(n=1.259)$ and during puerperium (upto 1 week; $n=654$ ). Next to the blood count, hemorheological parameters including red blood cell (RBC) -aggregation (stasis E0; low shear E1), -deformability (low, moderate and high shear conditions) and plasma viscosity (pv) were assessed. Plasma viscosity (pv) was examined using KSPV 1 Fresenius, RBC aggregation (stasis: E0 and low shear: E1) using MA1-Aggregometer; Myrenne and RBC deformability (def) was determined by Rheodyn SSD Diffractometer, Myrenne, Roetgen, Germany were tested. In some of these women laboratory results prior to pregnancy $(n=145)$ were available which were compared with those during pregnancy.

RESULTS: Mean maternal pv remained unchanged within each trimester and compared to the values before pregnancy and during early puerperium (Range of means: $1.18-1.20 \mathrm{mPa} \mathrm{S}$ ). In contrast, RBC agg (E0 and E1) was markedly higher in the 2 nd $(21.8 \pm 7.0$ and $28.9 \pm 9.4 ; p<0.001)$ and 3 rd trimester $(18.74 \pm 8.4$ and $28.2 \pm 9.4 ; p<0.01)$ compared to the values before pregnancy $(16.4 \pm 6.4$ and $20 \pm 7.5)$ and during 1 st trimester (17.49 \pm 6.5 and $22.4 \pm 7.4)$. There was a stat. significant temporary reduction in RBC def. under all shear rate conditions during 2nd trimester compared to the values before pregnancy which remained significantly lower during 3rd trimester only under high shear rates.

An increase RBC agg was stat. significantly inversely correlated with reduced RBC def being most pronounced under low shear rate conditions. While RBC rigidity was stat. significantly correlated with higher hematocrit values there was only a weak correlation between RBC agg and haematocrit (E0: $r=-0.084 ; p=0.03 ; \mathrm{E} 1: r=-0.06 ; p=0.1)$. Pv was not correlated with haematocrit or RBC def but stat. significantly correlated with RBC agg.

CONCLUSION: Blood rheological changes manifest during 1st trimester, and fairly remain unchanged during 2nd trimester until term. Physiologic hemodilution and increasing hypercoagulability is accompanied by high RBC -aggregation and -rigidity during 2nd trimester while plasma viscosity remains nearly unaffected throughout normal pregnancy.
\end{abstract}

Keywords: Normal pregnancy, haemorheology, red blood cell aggregation, red blood cell deformability

\section{Introduction}

Beginning at the 6th gestational week, continuous plasma volume expansion develops which is most pronounced at the end of the 2 nd trimester but in average remains higher in the range of $\sim 1.12 \mathrm{~L}$ at term as compared to the 1st trimester [1]. In addition, the contemporary rise in RBCs of 250 to $450 \mathrm{~mL}$ - predominantly driven by increased erythropoietin concentrations - results in a 30 to $50 \%$ increase of maternal blood volume compared to the non-pregnant state [2, 3]. Hemodilution is the physical

\footnotetext{
${ }^{*}$ Corresponding author: Prof. Dr. Georg-Friedrich von Tempelhoff, FCATH, Department of Obstetrics and Gynecology, Vinzenz Hospital of Hanau, Germany. Tel.: +49 6181272 371; E-mail: g-f.von.tempelhoff@ gmx.de.
} 
consequence of the disproportional increase in plasma volume and RBCs mass, whereas hemoglobin concentrations between 11.0-12.0 g/dL early during 3rd trimester are commonly present [4]. Although such hemoglobin values refer to anemia outside pregnancy, low hemoglobin concentrations associated with physiologic hemodilution have been linked to a more favorable outcome of pregnancy [5] while failure of this process signaled poor prognosis and was found to precede a higher complication rate such as pre-eclampsia [6], growth retarded fetus [7] and low fetal birth weight [8].

Undisputedly, the increased circulating volume usually allows blood loss during delivery upto $1.5 \mathrm{~L}$ in normovolemic healthy women without leading to maternal hemodynamic instability. Moreover, it has been speculated that both, reduced blood viscosity and increased circulating red blood cell mass associated with physiological hemodilution may improve blood flow at the materno-fetal surface thus guaranteeing optimal uterine and intervillous perfusion and finally oxygenation/supply to the fetus. In contrast, high maternal hemoglobin concentrations in the absence of physiologic hemodilution were found to coincide with thrombotic occlusions in the placental vasculature [9] which in turn were associated with high risk of intrauterine growth restriction [10].

Hypercoagulability is a further physiologic and progressive process during normal pregnancy [11]. Platelet aggregation and interaction of increased plasma protein concentrations associated with blood coagulation activation results in entrapment of red blood cells and clot formation which increases blood viscosity [12]. Under low shear rate conditions of the large vessels blood viscosity is mostly dependent on the number of erythrocytes [13] while in the high shear rate environment of the microcirculation, hematocrit is constantly reduced to values between 10 to $20 \%$ [14] in capillary vessels with diameter between 5 and $15 \mu \mathrm{m}$ (Fahraeus-Lindqvist effect). In such narrow vessels the composition of plasma and aggregability of red blood cells together with their shear force driven viscoelastic properties gain influence on local blood viscosity [15] which seems to be most significant under pathologic conditions e.g. sickle cell anemia associated with increased RBC -aggregation and/or reduced -deformability [16-18].

Results of blood rheological studies in pregnancy indicate significant differences regarding rheological properties of red blood cells and plasma viscosity compared to non-pregnant women towards an increase in blood viscosity. However, most of these findings were either restricted to a certain time interval during pregnancy e.g. 1st, 2nd, 3rd trimester, term or mostly included only small numbers of patients [19-24].

In order to analyze the dynamic of blood rheological properties we longitudinally assessed rheological variables throughout normal pregnancy and during early puerperium in an observational two center trial.

\section{Patients and methods}

In this two-centre trial, recruitment of women was performed from January 2013 to the end of December 2016. Women who visited the Department of Obstet. Gynecol. City Hospital of Aschaffenburg or the Department of Obstet. Gynecol. St. Vinzenz Hospital Hanau prior to or during their pregnancy and delivery were ask to participate in this non interventional trial. A careful medical history was taken prior to inclusion. Women were eligible for participating if they were otherwise healthy without chronic or acute diseases and not receiving permanent medication or anticoagulants, aspirin or any kind of antiplatelet drugs. Only singleton and uneventful pregnancies were eligible for final evaluation. Patients with pregnancy related morbidity and complications like preterm birth $(<37 \mathrm{GW})$, fetal birth-weight less than $2.500 \mathrm{~g}$ (low-birth weight new-born), fetal growth below $5 \%$ percentile of normal (IUGR: intrauterine growth restriction), pre-eclampsia (blood pressure equal to or more than $140 \mathrm{mmHg}$ systolic or $90 \mathrm{mmHg}$ diastolic in addition to proteinuria of more than $300 \mathrm{mg} / 24 \mathrm{~h}$ ), HELLP Syndrome and severe anaemia $(<9 \mathrm{~g} / \mathrm{dL})$ were excluded from the trial. 
Some data of the maternal log (Mutterpass) including personal, medical and social history, maternal age and body mass index (BMI), smoking, iron supplementation were anonymously obtained and considered for the results of blood rheological examinations.

Prior to the written informed consent given by all patients, it was made clear that results of this evaluation were without clinical consequence. A clearance certificate of this trial was granted by the ethical committee of the University of Würzburg, Germany.

Blood was drawn at all times during pregnancy until end of the 1 st week of puerperium. Some of the participants also had blood sampling prior to their pregnancy when they visited for routine care. The latter was termed "before pregnancy". Rheological results were calculated according to the gestational week (GW), within each trimester (1st Trim: < $13 \mathrm{GW}$; 2nd Trim: >12 GW and < 29th GW; IIIrd Trim: $>28$ th GW) and one week after delivery (puerperium).

Blood was drawn after minimal stasis of the upper arm from the antecubital vein using a 20 gauge needle. Blood was collected in vacuum tubes containing $1: 10$ potassium ethylene diamine tetraacetic acid (EDTA) and rheological estimations were immediately performed in compliance to ICSH guidelines/International Committee for Standardization in Haematology [25]. RBC aggregation was estimated using a photometric rheoscop developed by Schmid-Schoenbein et al./MA1-Aggregometer; Myrenne, Roetgen, Germany/. Blood samples adjusted to a standard haematocrit of $45 \%$ were placed between a transparent cone-plate system and rotated for 10 seconds at a high shear rate of $600 \mathrm{~s}^{-1}$ in order to disperse all pre-existing cell aggregates. Average red blood cell (RBC) aggregation was determined by the quantity of light transmission which is measured by photo sensors in two modes - during stasis - and while samples are subjected to low shear rate of $3 \mathrm{~s}^{-1}$. Light transmission increases proportionally with extend of RBC aggregation whereas processed data were expressed in arbitrary units. For determination of $\mathrm{Pv}$ vacuum tubes were centrifuged for 20 minutes $/ 2000 \mathrm{~g}$ at $4{ }^{\circ} \mathrm{C} /$, probes from the middle-layer of the plasma were obtained and inserted into the Capillary tube viscosimeter/KSPV 1 Fresenius, Bad Homburg Germany/at $37^{\circ} \mathrm{C}$.

\subsection{Statistics}

Descriptive analysis in the Tables and Figures are presented as mean values, standard deviations, medians; inter quartile ranges and 95 percent confidence intervals (Box Whisker Plots). In the majority of patients blood sampling was performed once. Therefor laboratory results at each estimation timepoint were handled as unrelated/independent samples. Homogeneity of Variance was assessed using Levene test. Because of unequal variances and sample sizes, Games-Howell test was used for post hoc analysis comparing laboratory results at each estimation time-point.

Correlation coefficients according to Spearman were calculated. Two sided $p$ values of less than 0.05 were considered statistical significant.

\section{Results}

This is a cross-sectional bicentral observational study. During the study period from January 2013 until December 2014 and from July 2015 until December 2016 a total of 945 women with singleton pregnancy agreed to participate in this non interventional trial. Blood was collected whenever these women visit one of the gynecologic units. During pregnancy $75 \%(n=715)$ of the women were by tested once, $15.4 \%(n=146)$ twice and $8.9 \%(n=84)$ had three estimation time-points for blood rheological variables. The distribution of blood sampling within each trimester was $250(19.9 \%)$ during the 1 st, 381 during the 2nd (30.3\%) and 628 (49.9\%) during the 3rd trimester. Hundred-and-forty-five of these women were tested prior to pregnancy and in 628 blood rheological estimations were performed 


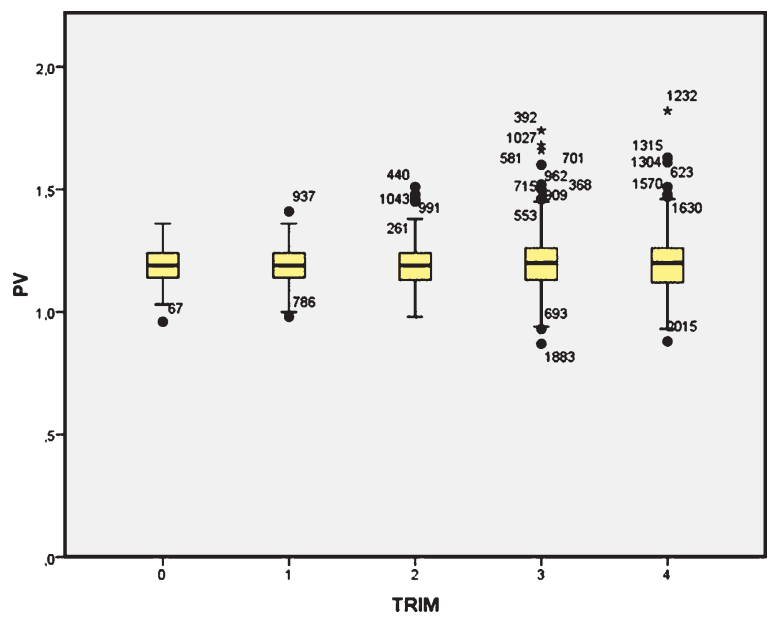

$0=$ prior to pregnancy $1=$ during $1^{\text {st }}$ Trimester $2=$ during $2^{\text {nd }}$ Trimester $3=$ during $3^{\text {rd }}$ Trimester $4=$ within $1^{\text {st }}$ week of puerperium

during the 1 st week of their puerperium. Mean age was $33.8(18-46 \mathrm{y})$ and BMI was $28.1 \mathrm{Kg} / \mathrm{m}^{2}$ $\left(17-50.3 \mathrm{~kg} / \mathrm{m}^{2}\right)$.

\section{Blood rheological results}

\subsection{Plasma viscosity}

Mean maternal pv (Fig. 1) remained unchanged within each trimester (Range of means: $1.19 \pm 0.07 \mathrm{mPas}$ and $1.20 \pm 0.11 \mathrm{mPas}$ ) and when compared with the mean values of women before pregnancy $(1.19 \pm 0.06 \mathrm{mPas} ; p=0.89)$ and during puerperium $(1.19 \pm 0.11 \mathrm{mPas} ; p=0.91)$. During pregnancy there was a stat. significant correlation between PV and low shear RBC aggregation $(r=0.12$; $p<0.001)$ but other than that no correlation was found including haematocrit $(r=0.04 ; p=0.3)$ and RBC deformabilities $(r<0.05 ; p>0.15)$.

\subsection{RBC aggregation (stasis and low shear)}

While mean RBC agg. in stasis and low shear (Fig. 2a) were barely unchanged during 1st trimester compared to the values before pregnancy (RBC agg stasis: $17.49 \pm 6.5$ vs. $16.37 \pm 6.5$; RBC agg low shear: $22.4 \pm 7.4$ vs. $20.8 \pm 7.5$ ), a stat. significant rise was found in the 2 nd and 3 rd trimester (RBC stasis: $21.8 \pm 7.0$ and $18.7 \pm 8.4 ; p<0.001$; RBC agg. low shear: $28.9 \pm 8.2$ and $28.2 \pm 9.4$; $p<0.001$ ) when compared with the values of the 1 st trimester and before pregnancy. RBC agg. remained stat significantly higher during early puerperium (RBC stasis: $20.5 \pm 7.1$ and RBC agg. low shear: $29.6 \pm 9.1 ; p<0.001)$.

Correlation coefficients were calculated for low shear RBC agg. and gestational weeks within each trimester and again when 1st and 2nd as well as 2nd and 3rd trimester were combined (Fig. 2b). While during 1st trimester there was a slight but stat. significant increase in low shear RBC agg. towards the 12th gestational week $(\mathrm{GW})$, values werer nearly unchanged during the 2nd trimester (GW 13 to 28) and were found to be stat. significantly inverse correlated with GW during 3rd trimester 


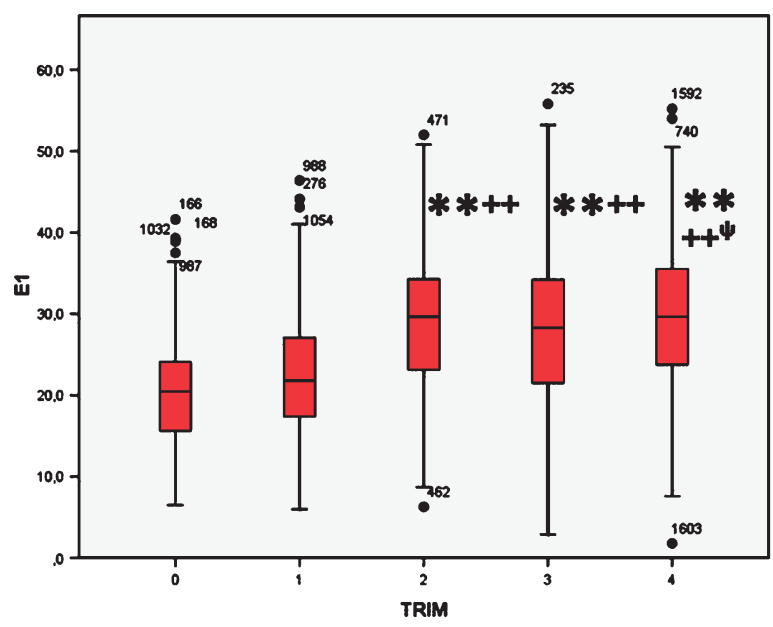

$0=$ prior to pregnancy $1=$ during $1^{\text {st }}$ Trimester $2=$ during $2^{\text {nd }}$ Trimester $3=$ during $3^{\text {rd }}$ Trimester $4=$ within $1^{\text {st }}$ week of puerperium

Fig. 2a. Box Whisker Plots of maternal Red Blood Cell aggregation under low shear conditions before, during normal pregnancy and puerperium. Median, interquartile range, minimum/maximum and extreme values (numbers) *: vs. before pregnancy: $p<0.05 ; * *: p<0.001 ;+$ : vs. first trimester: $p<0.05 ;++: p<0.001$; \#: vs. second trimester: $p<0.05$;\#\#: $p<0.001$; $\Psi$ : vs third trimester: $p<0.05 ; \Psi \Psi: p<0.001$.

\section{Spearman two-tailed correlation}
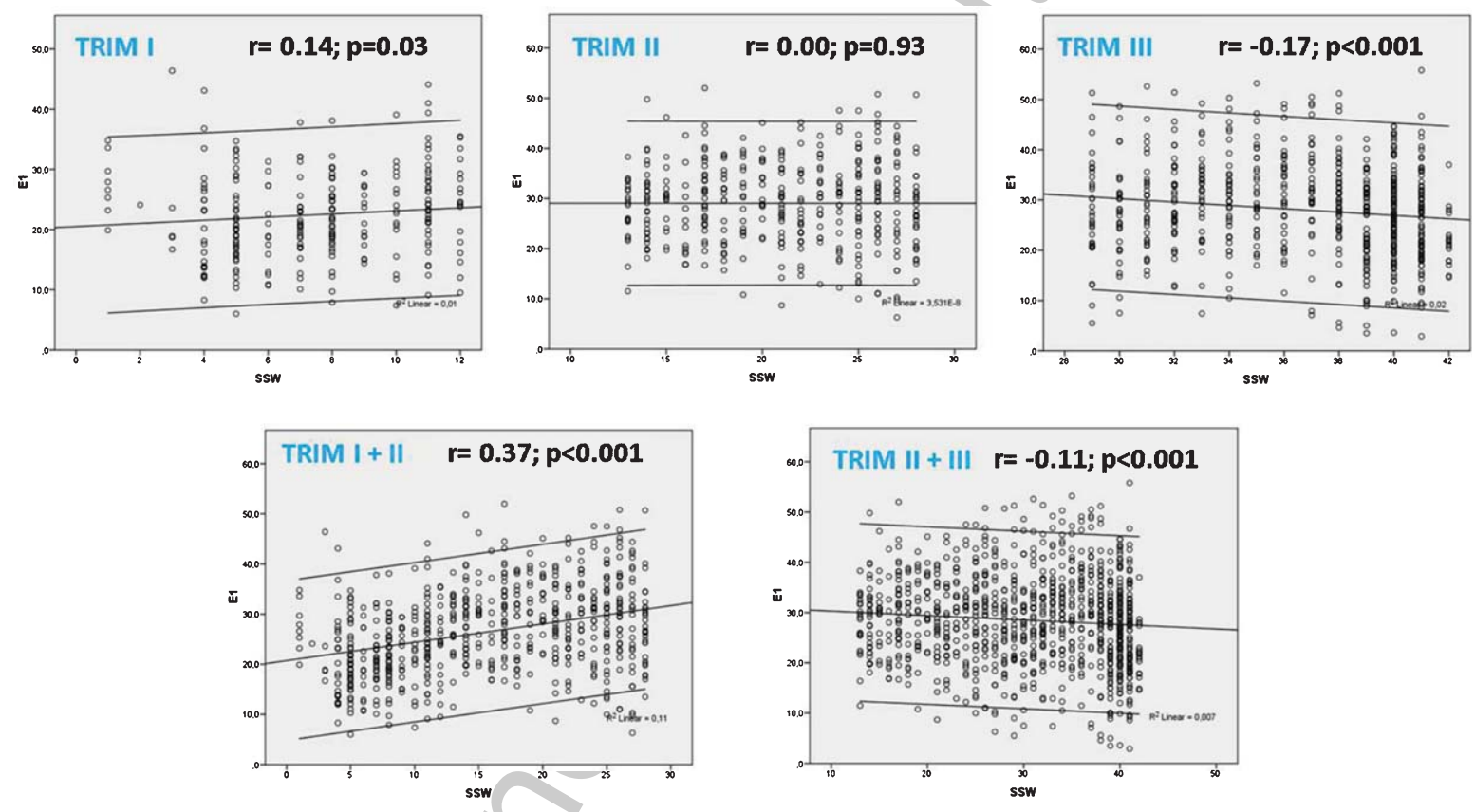

Fig. 2b. Correlation between low shear Red Blood Cell aggregation and gestational age. Spearman Correlation coefficient (two-sided). Correlations were tested within each Trimester (Trim I: 1st Trimester = until gestational week 13; Trim II: 2nd Trimester $=$ gestational week 14 to 28 , and Trim III: 3rd Trimester $=$ gestational week 29 until delivery) and within 1 st and 2nd Trimester and again within 2 nd and 3rd Trimester during pregnancy. $r=$ correlation coefficient; $p$-value.

(GW 29 to 42). Combining the 1st and 2nd trimester low shear RBC agg was stat. significantly correlated with GW while during 2 nd and 3rd trimester the correlation was stat. significantly inverse to the GW. 
Table 1

Red Blood Cell deformability before, during pregnancy and during early puerperium

\begin{tabular}{lccccc}
\hline & Before pregnancy & Trim I & Trim II & Trim III & Puerperium \\
\hline RBC def (1.2) low shear & $10.1 \pm 3.2$ & $9.5 \pm 3.3$ & $8.6 \pm 2.9^{* *,+}$ & $10.2 \pm 3.5^{\# \#}$ & $10.7 \pm 3.1^{++, \# \#}$ \\
RBC def (3.0) low shear & $21.0 \pm 4.7$ & $20.6 \pm 4.8$ & $19.2 \pm 4.4^{*,+}$ & $21.0 \pm 4.3^{\# \#}$ & $21.6 \pm 3.9^{+, \# \#}$ \\
RBC def (6.0) moderate shear & $29.8 \pm 5.2$ & $29.0 \pm 5.2$ & $27.5 \pm 5.0^{* *++}$ & $28.9 \pm 4.3^{\# \#}$ & $30.1 \pm 12.6^{\# \#}$ \\
RBC def (12.0) moderate shear & $36.0 \pm 5.7$ & $35.4 \pm 5.6$ & $33.8 \pm 5.7^{*,+}$ & $35.0 \pm 4.3^{\#}$ & $35.9 \pm 4.2^{\# \#, \Psi}$ \\
RBC def (30.0) high shear & $43.1 \pm 6.4$ & $42.2 \pm 6.1$ & $40.3 \pm 6.6^{*,+}$ & $41.2 \pm 4.4^{*}$ & $42.1 \pm 4.6^{\# \#, \Psi}$ \\
RBC def (60.0) high shear & $46.2 \pm 6.9$ & $45.4 \pm 6.9$ & $43.7 \pm 7.1^{*,+}$ & $44.4 \pm 4.8$ & $45.5 \pm 4.8^{\# \#, \Psi}$ \\
\hline
\end{tabular}

*: vs. before pregnancy: $p<0.05$; **: $p<0.001$; + : vs. first trimester: $p<0.05 ;++: p<0.001$; \#: vs. second trimester: $p<0.05$; \#\#: $p<0.001$. $\Psi$ : vs third trimester: $p<0.05 ; \Psi \Psi: p<0.001$.

\subsection{RBC deformability (under low, moderate and high shear conditions)}

Results of RBC deformability are summarized in Table 1 . While in the 1st trimester RBC deformability under all shear stress conditions was unchanged compared to the values before pregnancy there was a stat. significant reduction in the 2 nd trimester compared to that found before pregnancy and during 1 st trimester. Compared to the results during 2nd trimester, RBC def. under low shear conditions stat. significantly increased to levels of the initial values before pregnancy and during 1st trimester and remained stat. significantly higher after delivery (Fig. 3a). Moderate shear stress was associated with a constant and stat. significant increase in RBC def. in the 3rd trimester compared to the 2nd trimester and again during puerperium compared to the 3rd trimester (Fig. 4a). In contrast, RBC def. under high shear conditions were and remained stat. significant lower during 2nd and again in the 3rd trimester compared to the values before pregnancy. However, during early puerperium values were stat. significantly higher compared to those during 2nd and 3rd trimester (Fig. 5a). RBC def. under all shear forces were stat. significant inversely correlated with RBC aggregation during pregnancy as well as each trimester which was most pronounced in the presence of low shear $(r=-0.382 ; p<0.001)$ compared to high shear forces $(r=-0.205 ; p=0.002)$. RBC def. under all shear forces were stat. significant inversely correlated with GW during the 1st trimester while values were barely unchanged during 2 nd trimester and showed stat. significant increase deformability towards term in the 3rd trimester (Fig. 3b, $4 b$ and $5 b)$.

\subsection{Blood rheology and haematocrit}

Estimations of haematocrit and haemoglobin were available in 281 pregnant women. During pregnancy mean haematocrit was stat. significantly lower in the 1 st trimester $(37.4 \pm 24 \%)$ compared to non-pregnant women $(39.0 \pm 2.7 \%)$ and again in the 2 nd trimester $(34.4 \pm 3.0 \%)$ compared to the $1 \mathrm{st}$ trimester. Thereafter an increase was found during 3rd trimester $(35.6 \pm 2.9 \%)$ whereas mean values remained stat. significantly lower compared to the results in non-pregnant women. Accordingly we observed a stat. significant inverse correlation between haematocrit and haemoglobin as well and gestational age. There was no association between $\mathrm{Pv}$ and haematocrit or haemoglobin concentrations during neither early, nor late pregnancy. Haematocrit was stat. significantly correlated with RBC def. under all shear stress conditions at all times during pregnancy. While during pregnancy as a hole a weak inverse correlation between increasing RBC agg and lower haematocrits was found, this trend was no longer stat. significant when differentiating into early and late gestational age (Table 2). The course of pregnancy was characterized by continues increase in mean RBC agg. and a temporary reduction 


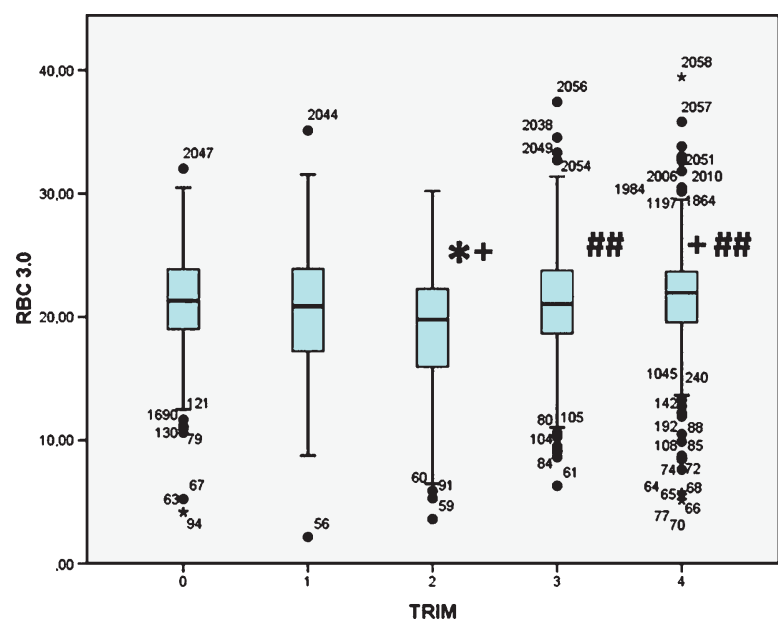

$0=$ prior to pregnancy

$1=$ during $1^{\text {st }}$ Trimester

$2=$ during $2^{\text {nd }}$ Trimester

$3=$ during $3^{\text {rd }}$ Trimester

$4=$ within $1^{\text {st }}$ week of puerperium

Fig. 3a. Box Whisker Plots of maternal Red Blood Cell deformability under low shear conditions before, during normal pregnancy and puerperium. Median, interquartile range, minimum/maximum and extreme values (numbers); *: vs. before pregnancy: $p<0.05$; **: $p<0.001 ;+$ : vs. first trimester: $p<0.05 ;++: p<0.001$; \#: vs. second trimester: $p<0.05$; \#\#: $p<0.001$ $\Psi$ : vs third trimester: $p<0.05 ; \Psi \Psi: p<0.001$.
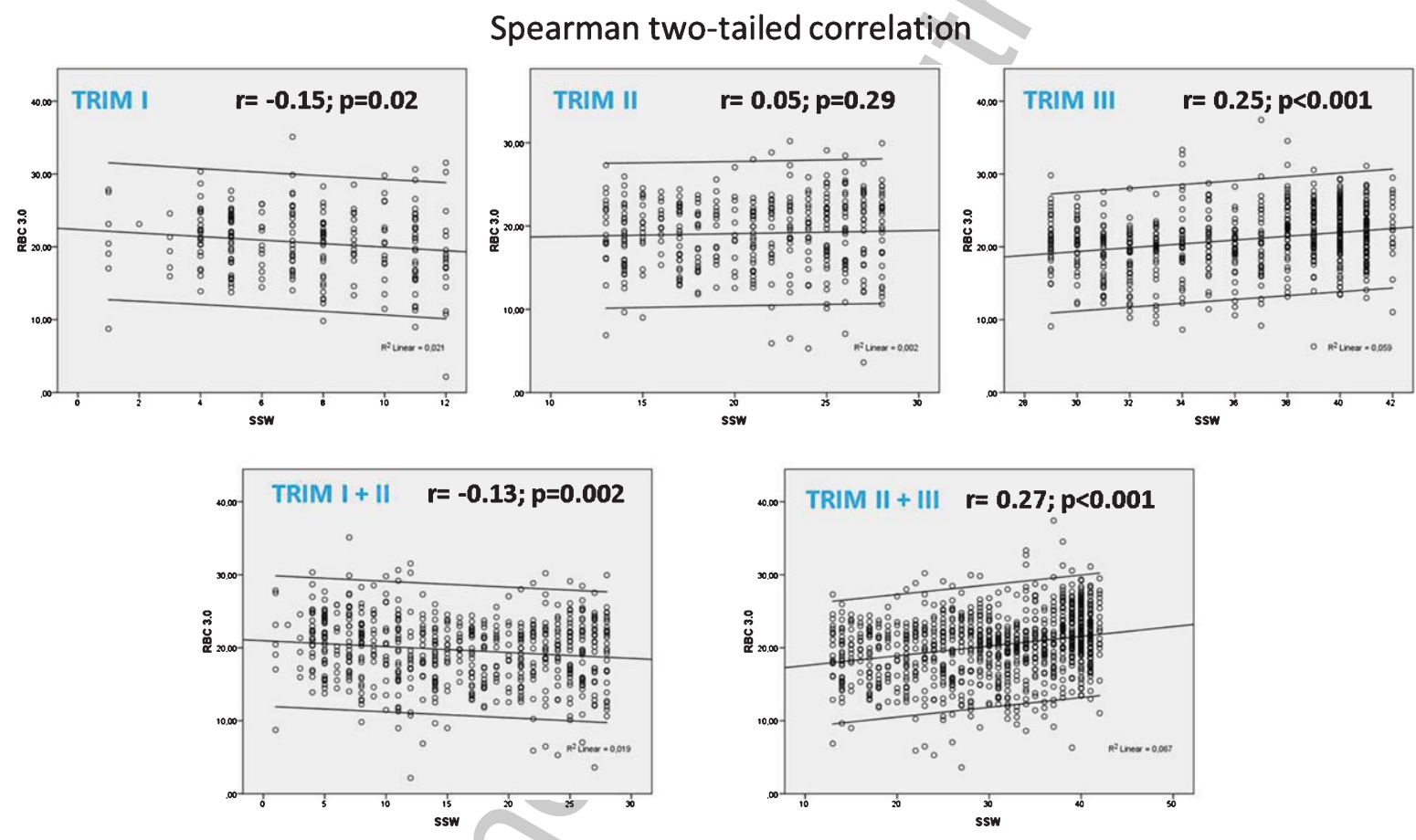

Fig. 3b. Correlation between low shear (3.0) Red Blood Cell deformability and gestational age. Spearman Correlation coefficient (two-sided). Correlations were tested within each Trimester (Trim I: 1st Trimester $=$ until gestational week 13; Trim II: 2nd Trimester = gestational week 14 to 28, and Trim III: 3rd Trimester=gestational week 29 until delivery) and within 1 st and 2 nd Trimester and again within 2 nd and 3 rd Trimester during pregnancy. $r=$ correlation coefficient; $p$-value.

of mean RBC def. and mean haematocrit as well which was most pronounced during 2nd trimester and which was followed by only a moderate tendency of normalization towards the end of pregnancy. Plasma viscosity remained unchanged throughout normal pregnancy (Fig. 6). 


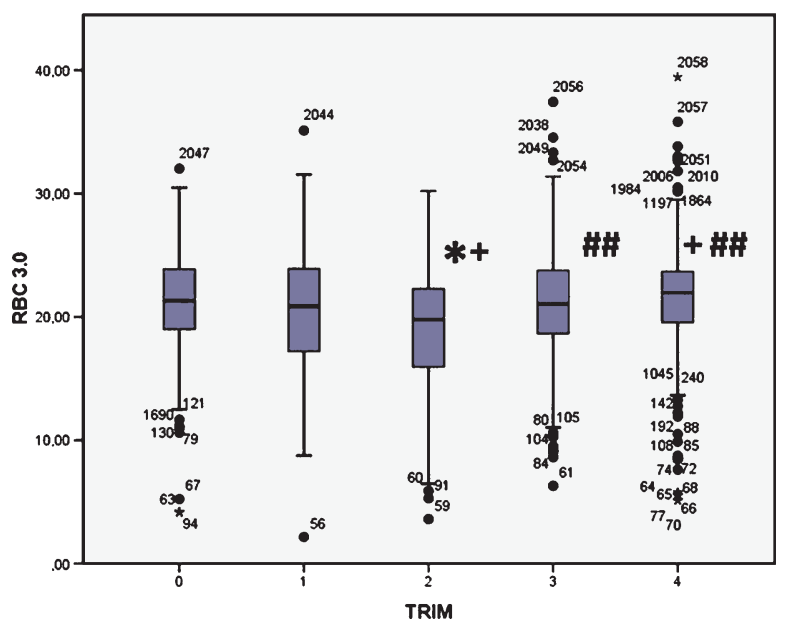

$0=$ prior to pregnancy $1=$ during $1^{\text {st }}$ Trimester 2 = during $2^{\text {nd }}$ Trimester $3=$ during $3^{\text {rd }}$ Trimester $4=$ within $1^{\text {st }}$ week of puerperium

Fig. 4a. Box Whisker Plots of maternal Red Blood Cell deformability under moderate shear conditions before, during normal pregnancy and puerperium. Median, interquartile range, minimum/maximum and extreme values (numbers) *: vs. before pregnancy: $p<0.05 ; * *: p<0.001 ;+$ : vs. first trimester: $p<0.05 ;++: p<0.001$; \#: vs. second trimester: $p<0.05$; \#\#: $p<0.001$; $\Psi$ : vs third trimester: $p<0.05 ; \Psi \Psi: p<0.001$.

\section{Spearman two-tailed correlation}
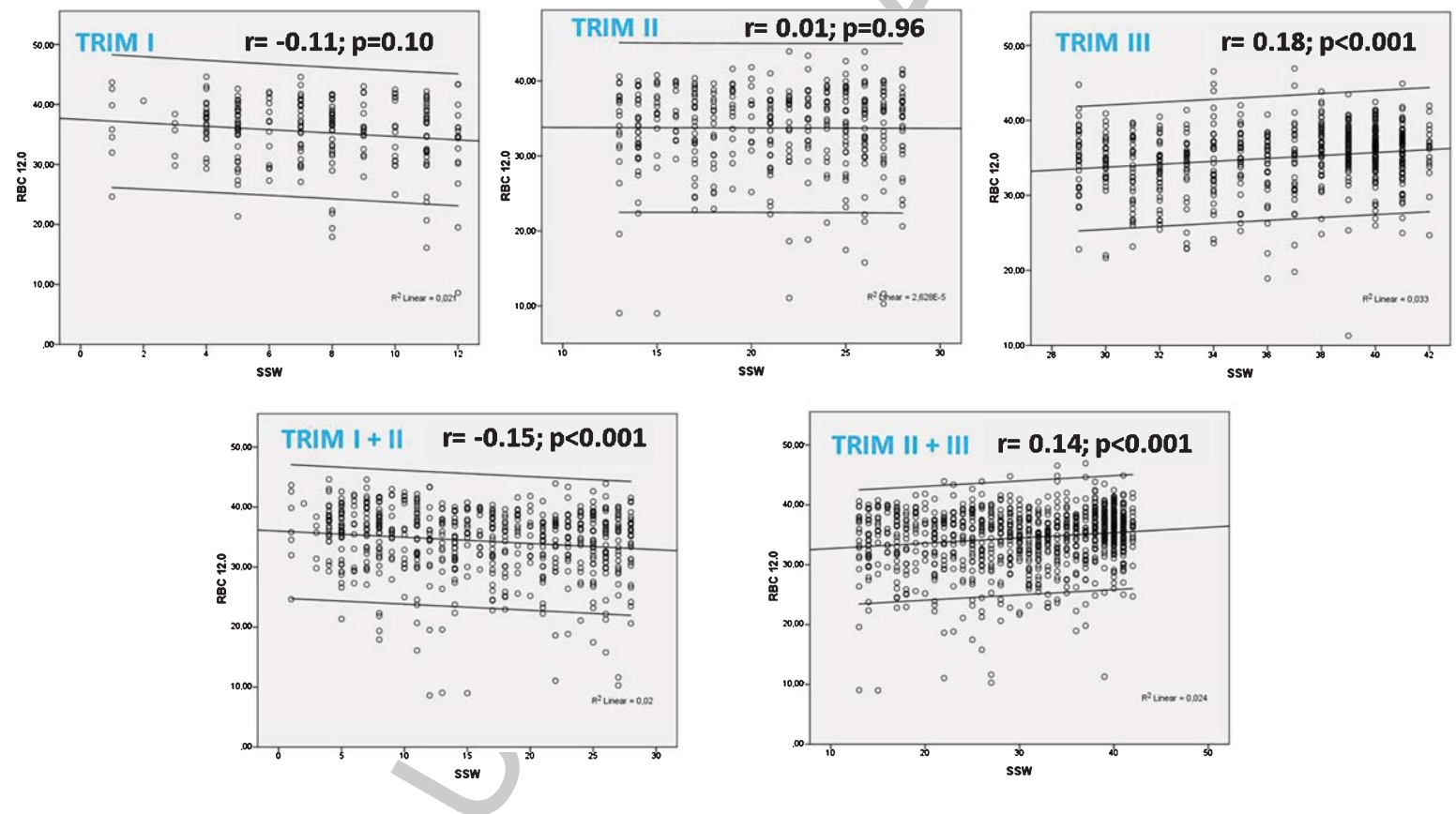

Fig. 4b. Correlation between moderate (12.0) shear Red Blood Cell deformability and gestational age. Spearman Correlation coefficient (two-sided). Correlations were tested within each Trimester (Trim I: 1st Trimester = until gestational week 13; Trim II: 2nd Trimester = gestational week 14 to 28, and Trim III: 3rd Trimester = gestational week 29 until delivery) and within 1 st and 2 nd Trimester and again within 2 nd and 3rd Trimester during pregnancy. $r=$ correlation coefficient; $p$-value. 


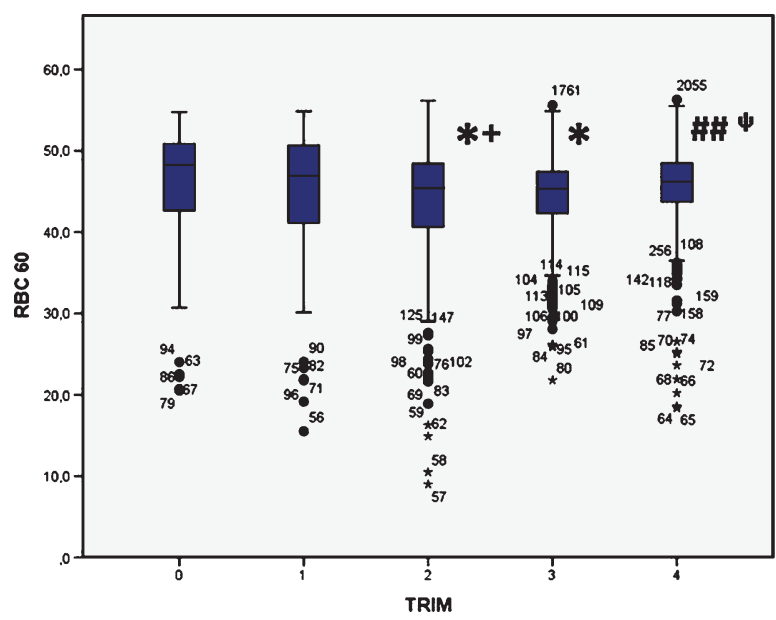

$0=$ prior to pregnancy

$1=$ during $1^{\text {st }}$ Trimester

$2=$ during $2^{\text {nd }}$ Trimester

$3=$ during $3^{\text {rd }}$ Trimester

$4=$ within $1^{\text {st }}$ week of puerperium

Fig. 5a. Box Whisker Plots of maternal Red Blood Cell deformability under high shear conditions before, during normal pregnancy and puerperium. Median, interquartile range, minimum/maximum and extreme values (numbers) *: vs. before pregnancy: $p<0.05 ; * *: p<0.001 ;+$ : vs. first trimester: $p<0.05 ;++: p<0.001$; \#: vs. second trimester: $p<0.05$; \#\#: $p<0.001$; $\Psi$ : vs third trimester: $p<0.05 ; \Psi \Psi: p<0.001$.

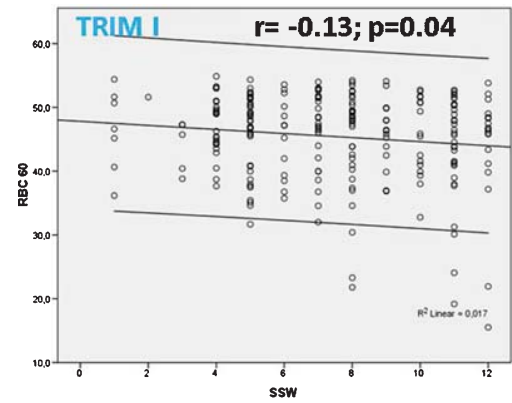

Pearson two-tailed correlation
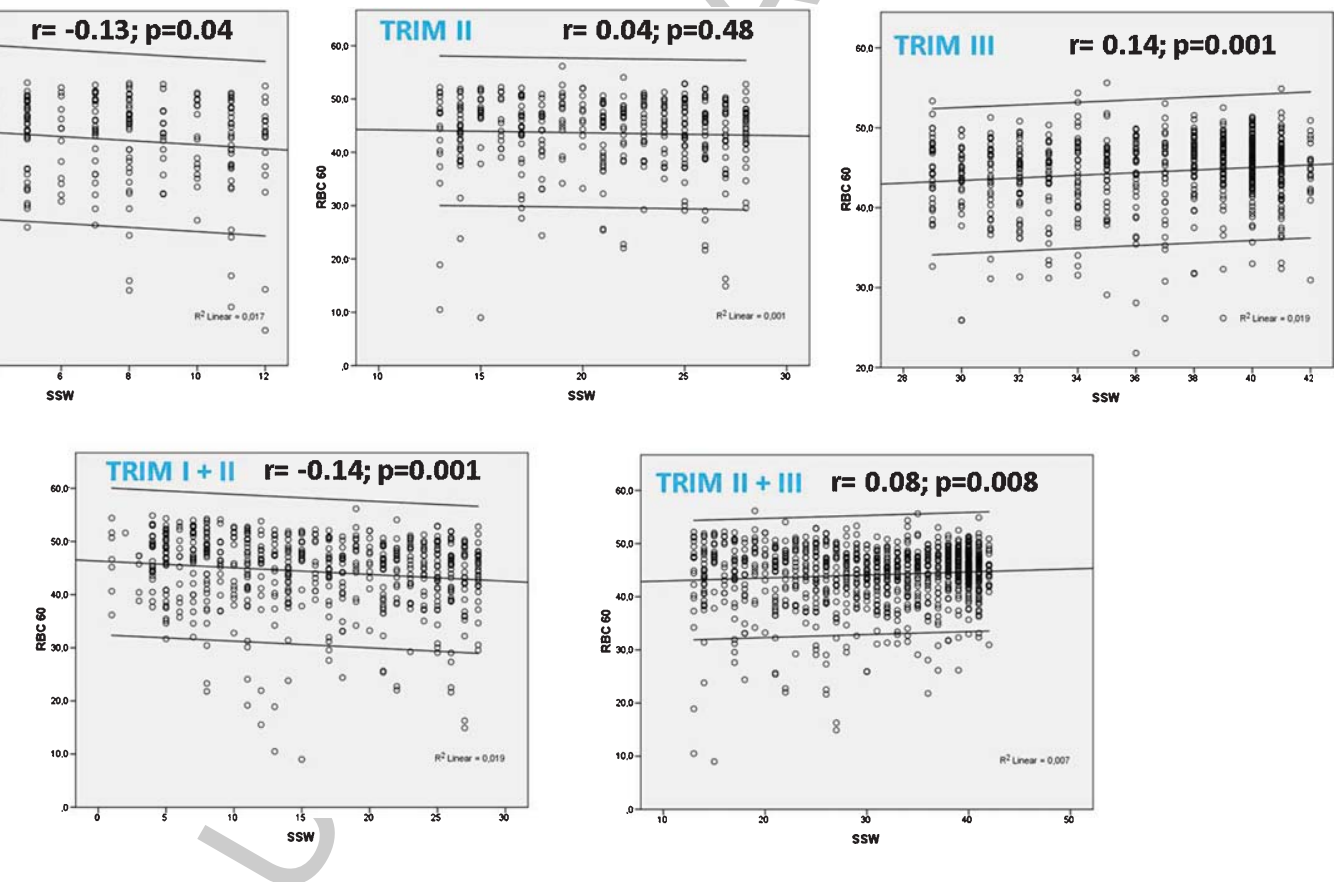

Fig. 5b. Correlation between high (60.0) shear Red Blood Cell deformability and gestational age. Spearman Correlation coefficient (two-sided). Correlations were tested within each Trimester (Trim I: 1st Trimester = until gestational week 13; Trim II: 2nd Trimester = gestational week 14 to 28, and Trim III: 3rd Trimester = gestational week 29 until delivery) and within 1 st and 2 nd Trimester and again within 2 nd and 3rd Trimester during pregnancy. $r=$ correlation coefficient; $p$-value. 
Table 2

Correlation between Blood rheological parameters, gestational week and hematocrit/hemoglobin during two intervals; 1st and 2 nd and 2 nd and 3rd trimester of pregnancy

\begin{tabular}{|c|c|c|c|c|c|c|c|c|c|c|c|}
\hline \multicolumn{12}{|c|}{ Trimester I, II and III } \\
\hline & & GW & PV & Eo & E1 & $\mathrm{RBC} 1.2$ & RBC 3.0 & ORBC 6.0 & RBC 12.0 & RBC 30 & RBC 60 \\
\hline \multirow{3}{*}{$\begin{array}{l}\text { Gestational } \\
\text { week }(\mathrm{GW})\end{array}$} & Correlation koefficient & 1.000 & 0.050 & $-0.070^{*}$ & $0.123^{* *}$ & $0.202^{* *}$ & $0.153^{* *}$ & $0.077^{* *}$ & 0.021 & -0.056 & $-0.071^{*}$ \\
\hline & Sig. (2-sided) & - & 0.078 & 0.013 & 0.000 & 0.000 & 0.000 & 0.008 & 0.461 & 0.055 & 0.013 \\
\hline & $\mathrm{N}$ & 1259 & 1244 & 1249 & 1252 & 1189 & 1218 & 1188 & 1218 & 1189 & 1218 \\
\hline \multirow[t]{3}{*}{$\mathrm{Hb}$} & Correlation koefficient & $0.316^{* *}$ & $0.027-$ & $-0.119^{* *}$ & -0.058 & $0.091^{*}$ & $0.122^{* *}$ & $0.141^{* *}$ & $0.100^{*}$ & 0.052 & 0.048 \\
\hline & Sig. (2-sided) & 0.000 & 0.500 & 0.002 & 0.141 & 0.020 & 0.002 & 0.000 & 0.011 & 0.181 & 0.219 \\
\hline & $\mathrm{N}$ & 651 & 643 & 646 & 650 & 651 & 651 & 650 & 651 & 651 & 651 \\
\hline \multirow[t]{3}{*}{ Hct } & Correlation koefficient & $0.258^{* *}$ & 0.033 & $-0.084^{*}$ & -0.065 & $0.136^{* *}$ & $0.171^{* *}$ & $0.195^{* *}$ & $0.175^{* *}$ & $0.144^{* *}$ & $0.146^{* *}$ \\
\hline & Sig. $(2$ & 0.000 & 0.402 & 0.033 & 0.099 & 0.000 & 0.000 & 0.000 & 0.000 & 0.000 & 0.000 \\
\hline & $\mathrm{N}$ & 649 & 641 & 644 & 648 & 649 & 649 & 648 & 649 & 649 & 649 \\
\hline \multicolumn{12}{|c|}{ Trimester I and II ( $<29$ th Gestational week) } \\
\hline & & GW & PV & Eo & E1 & $\mathrm{RBC} 1.2$ & RBC 3.0 & RBC 6.01 & RBC 12.0 & RBC 30 & RBC 60 \\
\hline \multirow{3}{*}{$\begin{array}{l}\text { Gestational } \\
\text { week }(\mathrm{GW})\end{array}$} & Correlation koefficient & 1 & -0.064 & $0.238^{* *}$ & $0.332^{* *}$ & $-0.112^{* *}$ & $-0.138^{* *}$ & $-0.140^{* *}$ & $-0.142^{* *}$ & $-0.137^{* *}$ & * -0.139 ** \\
\hline & Sig. (2-sided) & - & 0.109 & 0.000 & 0.000 & 0.007 & 0.001 & 0.001 & 0.000 & 0.001 & 0.001 \\
\hline & $\mathrm{N}$ & 641 & 636 & 636 & 636 & 578 & 603 & 578 & 603 & 578 & 603 \\
\hline \multirow[t]{3}{*}{$\mathrm{Hb}$} & Correlation koefficient & $-0.318^{* *}$ & 0.021 & -0.075 & -0.119 & 0.009 & 0.070 & 0.123 & 0.136 & 0.144 & 0.149 \\
\hline & Sig. (2-sided) & 0.000 & 0.797 & 0.362 & 0.146 & 0.913 & 0.394 & 0.132 & 0.097 & 0.078 & 0.069 \\
\hline & $\mathrm{N}$ & 150 & 150 & 149 & 150 & 150 & 150 & 150 & 150 & 150 & 150 \\
\hline \multirow[t]{3}{*}{ Hct } & Correlation koefficient & $-0.390^{* *}$ & -0.008 & -0.029 & -0.123 & 0.042 & 0.110 & $0.170^{*}$ & $0.217^{* *}$ & $0.237^{* *}$ & $0.246^{* *}$ \\
\hline & Sig. (2-sided) & 0.000 & 0.925 & 0.728 & 0.137 & 0.615 & 0.181 & 0.039 & 0.008 & 0.004 & 0.003 \\
\hline & $\mathrm{N}$ & 148 & 148 & 147 & 148 & 148 & 148 & 148 & 148 & 148 & 148 \\
\hline \multicolumn{12}{|c|}{ Trimester II and III (>13th Gestational week) } \\
\hline & & GW & PV & Eo & E1 & RBC 1.2 & RBC 3.0 & RBC 6.01 & $\mathrm{RBC} 12.0$ & RBC 30 & $\mathrm{RBC} 60$ \\
\hline \multirow{3}{*}{$\begin{array}{l}\text { Gestational } \\
\text { week (GW) }\end{array}$} & Correlation koefficient & 1.000 & -0.058 & $0.259^{* *}$ & $0.337^{* *}$ & $-0.109^{* *}$ & $-0.127^{* *}$ & $-0.145^{* *}$ & $-0.145^{* *}$ & $-0.172^{* *}$ & * $-0.172^{* *}$ \\
\hline & Sig. (2-sided) & - & 0.144 & 0.000 & 0.000 & 0.009 & 0.002 & 0.000 & 0.000 & 0.000 & 0.000 \\
\hline & $\mathrm{N}$ & 641 & 636 & 636 & 636 & 578 & 603 & 578 & 603 & 578 & 603 \\
\hline \multirow[t]{3}{*}{$\mathrm{Hb}$} & Correlation koefficient & $-0.270^{* *}$ & -0.004 & -0.100 & -0.132 & 0.043 & 0.110 & $0.178^{*}$ & $0.162^{*}$ & 0.149 & 0.157 \\
\hline & Sig. (2-sided) & 0.001 & 0.963 & 0.225 & 0.107 & 0.602 & 0.179 & 0.029 & 0.048 & 0.069 & 0.056 \\
\hline & $\mathrm{N}$ & 150 & 150 & 149 & 150 & 150 & 150 & 150 & 150 & 150 & 150 \\
\hline \multirow[t]{3}{*}{ Hct } & Correlation koefficient & $-0.296^{* *}$ & 0.001 & -0.015 & -0.094 & 0.073 & 0.132 & $0.210^{*}$ & $0.245^{* *}$ & $0.247^{* *}$ & $0.267^{* *}$ \\
\hline & Sig. (2-sided) & 0.000 & 0.991 & 0.857 & 0.258 & 0.376 & 0.109 & 0.010 & 0.003 & 0.002 & 0.001 \\
\hline & $\mathrm{N}$ & 148 & 148 & 147 & 148 & 148 & 148 & 148 & 148 & 148 & 148 \\
\hline
\end{tabular}

\section{Discussion}

Significant hemostaseologic, hematologic and hemodynamic changes put women at higher risk for cardio vascular complications in upto $4 \%$ of pregnancies [26]. These changes potentially influence the blood rheological properties during pregnancy as well. Moreover, rheological changes were found to precede several severe obstetric complications, thus assessment of blood rheological variables may be helpful in identifying women at increased risk for such complications [2, 21, 23, 24, 31]. 

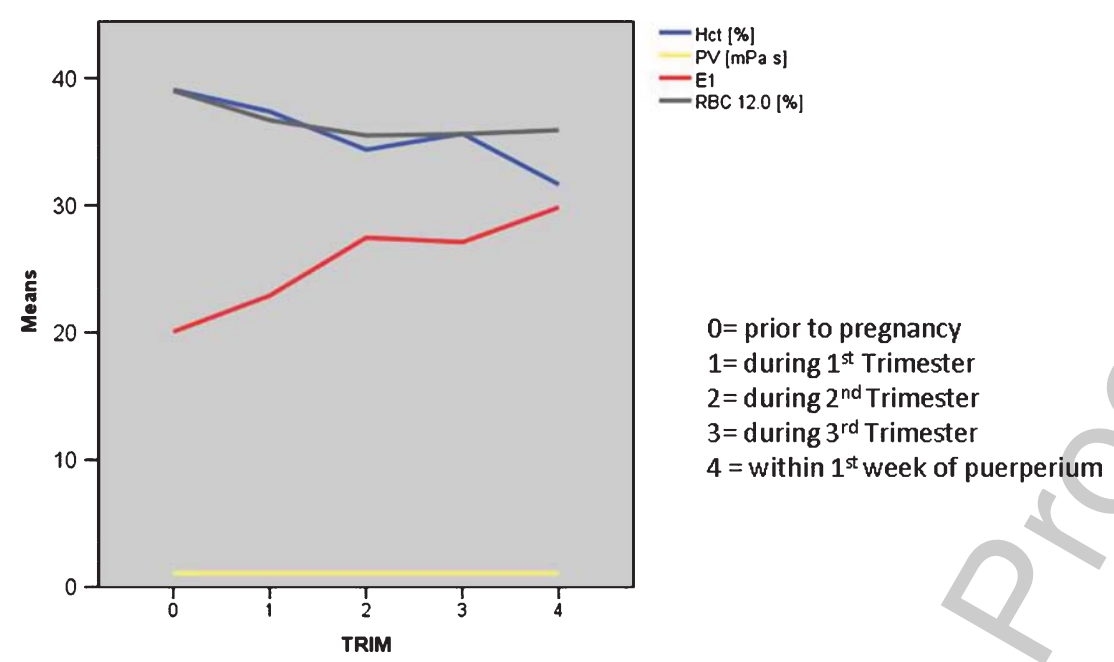

Fig. 6. Mean values of hematocrit, low shear RBC aggregation, RBC deformability under moderate shear forces and Plasma viscosity before pregnancy, within each trimester during pregnancy and within 1st week of puerperium.

Impaired blood flow at the utero placental cross-over in the presence of pathologic results of blood rheological parameters were considered possible triggers or symptoms of severe complications including recurrent miscarriage [27], pre-eclampsia [20, 24], pregnancy induced hypertension (PIH) [21], fetal growth restriction [23, 31], and preterm birth [31].

Blood rheological monitoring in 36 women with normal pregnancy revealed significantly inverse correlation between RBC rigidity and gestational age, fetal biometry assessed by ultrasound during GW 25 to 36, as well as the fetal birthweight and Apgar score. Plasma viscosity was only slightly but non-significantly increased during pregnancy. It was concluded that reduced RBC deformability may be a risk factor for impaired fetal growth, lower birthweight and lower gestational age at birth [23]. In a prospective trial by Robins et al. [21], mean RBC aggregation during 1st trimester in 579 pregnant women was significantly higher as compared to 213 non-pregnant healthy women while plasma viscosity was not significantly different in groups. During follow-up, among a subset of 248 pregnancies, 48 developed pregnancy induced hypertension $(\mathrm{PIH})$, whereas previous 1 st trimester plasma viscosity was moderately higher and RBC agg. was indifferent compared to women with uneventful pregnancy.

There are some remarkable findings in our trial regarding the timing of blood rheological changes in the course of normal pregnancy. Our data confirm a marked increase in RBC aggregation and contemporary reduction in RBC deformability until the beginning of the 2 nd trimester i.e. GW 13th which is accompanied by decreasing hematocrit concentrations. However, both of which remain nearly unchanged during 2nd trimester, while in the 3rd trimester RBC aggregation decreases and RBC deformability reestablishes during the same period. The significant correlation between RBC aggregation and rigidity at all times during pregnancy suggests that the limited deformability depends to a great extent on their tendency to form aggregates. During normal pregnancy the concentrations of most coagulation factors including fibrinogen dramatically increase above the normal range [11]. Although a statistically significant correlation between high fibrinogen concentration and $\mathrm{Pv}$ has been demonstrated in various pathologic conditions e.g. malignancy [28], coronary heart disease [29], vascular disease [30] and pregnancy as well [25], Pv seems unaffected by hyper coagulabilty and remains unchanged compared to non-pregnant women and throughout normal pregnancy. Likewise this is attributable to the diluting effect of plasma volume expansion on high coagulation factors concentrations. In a previous study, $\mathrm{Pv}$ at term was lower in women with complicated outcome of pregnancy and was inversely correlated with the number of pathologic coincidental conditions most likely as a result 
of renal protein loss e.g. in pre-eclampsia [31]. The significant increase in the 1st and persistent high extent in RBC aggregation during 2nd trimester found in our trial potentially blocks or at least slows down capillary perfusion as seen in various diseases associated with pathologic RBC aggregability [16]. Aggregation depends on the number and shape of the RBCs and increases in the presence of high plasma protein concentrations. In pregnancy, likewise high concentrations of coagulation factors including fibrin/fibrinogen split products, - the latter of which can bridge the RBCs via hydrogen bonds - represent a main cause of extensive RBC aggregation [32]. So far "impaired" RBC deformability apart from severe crisis of sickle cell anemia has not been proven to result in reduced blood flow velocities in the capillaries of the microcirculation. Although travel through capillary diameters smaller than that of the red blood cells should depend on their capacity of shear stress induced deformation, nail fold microscopy revealed indifferent blood velocities in adjacent capillaries of various calibers despite supply by the same vessel. Thus, endothelium itself seems capable synchronizing blood flow in the capillary network (reviewed in [15]).

While high shear forces in the microcirculatory capillaries sufficiently compensate and overcome rigidity of RBCs to pass through vessels, blood flow in the intervillious space may be more vulnerable due to its vascular architecture. During conversion of the maternal spiral arteries loss of smooth muscle and elastic lamina from the vessel wall until the inner third of the myometrium results in a 5-10-fold dilation at the vessel entrance. According to three-dimensional reconstructions models in which effects of terminal dilation on inflow of blood into the placental intervillous space was quantified dilation slows the rate of flow from 2 to $3 \mathrm{~m} / \mathrm{s}$ in the non-dilated part of the arteries (diameter: $0.4-0.5 \mathrm{~mm}$ ) to approximately $10 \mathrm{~cm} / \mathrm{s}$ at the entrance where the diameter is $\sim 2.5 \mathrm{~mm}$ [33]. Depending on the exact radius and viscosity a transit time through the intervillous space of approximately $25 \mathrm{~s}$ is assumed. The constellation of low shear stress in dilated areas in the presence of high RBC -aggregation and -rigidity potentially impact blood viscosity and flow velocity in the intervillous space. Reduced blood flow together with high coagulation activity caused by trophoblast cells expressed TF [34] may favor development of fibrin clots as seen in placental histology of pregnancies after delivery of growth retarded fetus [9].

Manifestation of blood rheological changes that take place during 1st trimester and fairly remain unchanged during 2 nd trimester is the most important finding of our current trial. We believe that inappropriate conversion of spiral arteries and trophoblast invasion are significant key features for subsequent pathologic obstetric conditions and in this context excessive changes of the blood rheologic properties in the 1st trimester may have unfavorable influence on the different stages of placentation.

\section{Acknowledgments}

This publication is dedicated to Prof. Dr. Friedrich Jung on the occasion of his 70th birthday. Detailed data of this manuscript are part of the thesis by Mrs. Barbara Niesigk.

\section{References}

[1] de Haas S, Ghossein-Doha C, van Kuijk SM, van Drongelen J, Spaanderman ME. Physiological adaptation of maternal plasma volume during pregnancy: A systematic review and meta-analysis. Ultrasound Obstet Gynecol. 2017;49:177-87.

[2] Hytten FE, Paintin DB. Increase in plasma volume during normal pregnancy. J Obstet Gynaecol Br Commonw. 1963;70 402-7.

[3] Feldschuh J, Enson Y. Prediction of the normal blood volume. Relation of blood volume to body habitus. Circulation. 1977;56:605-12. 
[4] Faupel-Badger JM, Hsieh CC, Troisi R, Lagiou P, Potischman N. Plasma volume expansion in pregnancy: Implications for biomarkers in population studies. Cancer Epidemiol Biomarkers Prev. 2007;16:1720-3.

[5] Little MP, Brocard P, Elliott P, Steer PJ. Hemoglobin concentration in pregnancy and perinatal mortality: A London-based cohort study. Am J Obstet Gynecol. 2005;193:220-6.

[6] Amburgey OA, Ing E, Badger GJ, Bernstein IM. Maternal hemoglobin concentration and its association with birth weight in newborns of mothers with preeclampsia. J Matern Fetal Neonatal Med. 2009;22:740-4.

[7] Blankson ML, Goldenberg RL, Cutter G, Cliver SP. The relationship between maternal hematocrit and pregnancy outcome: Black-white differences. J Natl Med Assoc. 1993;85:130-4.

[8] Jwa SC, Fujiwara T, Yamanobe Y, Kozuka K, Sago H. Changes in maternal hemoglobin during pregnancy and birth outcomes. BMC Pregnancy Childbirth. 2015;15:80-90.

[9] Bjerkedal T, Bakketeig L, Lehmann EH. Percentiles of birth weights of single, live births at different gestation periods. Acta Paediatr Scand. 1973;62:449-57.

[10] Lund CJ, Donovan JC. Blood volume during pregnancy. Significance of plasma and red cell volumes. Am J Obstet Gynecol. 1967;98:394-403.

[11] Prisco D, Ciuti G, Falciani M. Hemostatic changes in normal pregnancy. Haematologica Reports. 2005;1:1-5.

[12] James R. Byrnes and Alisa S. Wolberg. Red blood cells in thrombosis. Blood. 2017;130:1795-9.

[13] Pries AR, Neuhaus D, Gaehtgens P. Blood viscosity in tube flow: Dependence on diameter and hematocrit. Am J Physiol. 1992;263:H1770-8.

[14] Barras JP. Blood rheology - general review. Bibl Haematol.1969;33;277-97.

[15] Jung F, Ramplig M. Role of blood viscosity in the microcirculation. Clin Hemorheol Microcirc. 2016;64:251-4.

[16] Jung F, Mrowietz C, Hiebl B, Franke RP, Pindur G, Sternitzky R. Influence of rheological parameters on the velocity of erythrocytes passing nail fold capillaries in humans. Clin Hemorheol Microcirc. 2011:48,129-39.

[17] Renoux C, Romana M, Joly P, et al, Effect of age on blood rheology in sickle cell anaemia and sickle cell haemoglobin C disease: A cross-sectional study. PLoS ONE. 11(6):e0158182. doi:10.1371/journal.pone.0158182

[18] Chien S. Rheology in the microcirculation in normal and low flow states. Adv Shock Res. 1982;8:71-80.

[19] Csorba R, Soliman AA, Wieg C, Tsikouras P, Rath W, von Tempelhoff GF. Correlation of rheological parameters in maternal and fetal blood at term. J Matern Fetal Neonatal Med. 2015;28:969-76.

[20] Soliman AA, Csorba R, Yilmaz A, Tsikoaras P, von Tempelhoff GF. Rheologic results and their correlation to hemostatic changes in patients with moderate and severe preeclampsia: An observational cross-sectional study. Clin Hemorheol Microcirc. 2015;59:1-15.

[21] Robins JB, Woodward M, Lowe G, McCaul P, Cheyne H, Walker JJ. First trimester maternal blood rheology and pregnancy induced hypertension. J Obstet Gynaecol. 2005;25:746-50.

[22] Kametas N, Krampl E, McAuliffe F, Rampling MW, Nicolaides KH. Haemorheological adaptation during pregnancy in a Latin American population. Eur J Haematol. 2001;66:305-11.

[23] Martínez-Morales S, Bonillo-Perales A, Muñoz-Hoyos A, Puertas-Prieto A, Uberos-Fernández J, Molina-Carballo A, Bonillo-Perales JC, Sabatel-López R. The influence of maternal erythrocyte deformability on fetal growth, gestational age and birthweight. J Perinat Med. 1999;27:166-72.

[24] Heilmann L, Siekmann U, Schmid-Schönbein H, Ludwig H. Hemoconcentration and pre-eclampsia. Arch Gynecol. 1981;231:7-21.

[25] Bourner G, De la Salle B, George T, Tabe Y, Baum H, Culp N, Keng TB. ICSH guidelines for the verification and performance of automated cell counters for body fluids. International Committee for Standardization in Hematology (ICSH). Int J Lab Hematol. 2014;36:598-612.

[26] Regitz-Zagrosek V, Seeland U, Geibel-Zehender A, et al. Cardiovascular diseases in pregnancy. Dtsch Arztebl Int. 2011;108:267-73.

[27] Heilmann L, Kriechbaum A, Hojnacki B, Bode V, Wolf HZ. Clinical experiences with passive immunotherapy in habitual abortion. Geburtshilfe Perinatol. 1993;197:55-8.

[28] Tempelhoff G-F, Heilmann L, Pollow K, Hommel G. Monitoring of rheologic variables during postoperative high-dose brachytherapy for uterine cancer. Clin Appl Thromb Hemost. 2004;10:239-48.

[29] Leschke M. Rheological factors in coronary heart disease. Dtsch Med Wochenschr. 2008;133:270-3.

[30] Welsh P, Woodward M, Rumley A, Lowe G. Associations of plasma pro-inflammatory cytokines, fibrinogen, viscosity and C-reactive protein with cardiovascular risk factors and social deprivation: The fourth Glasgow MONICA study. Br J Haematol. 2008;141:852-61.

[31] von Tempelhoff G-F, Velten E, Yilmaz A, Hommel G, Heilmann L, Koscielny J. Blood rheology at term in normal pregnancy and in patients with adverse outcome events. Clin Hemorheol Microcirc. 2009;42:127-39.

[32] Rampling MW, Whittingstall MW, Linderkamp O. The effects of fibrinogen and its plasmin degradation products on the rheology of erythrocyte suspension. Clin Hemorheol Microcirc. 1984;4:533-41. 
[33] Burton GJ, Woods AW, Jauniaux E, Kingdom JC. Rheological and physiological consequences of conversion of the maternal spiral arteries for uteroplacental blood flow during human pregnancy. Placenta. 2009;30:473-82.

[34] Reverdiau P, Jarousseau AC, Thibault G, et al. Tissue factor activity of syncytiotrophoblast plasma membranes and tumoral trophoblast cells in culture. Thromb Haemost. 1995;73:49-54. 